\title{
Thyroid hormones: a potential ally to LDL-cholesterol-lowering agents
}

\author{
Leonidas H. Duntas, ${ }^{1}$ Gabriela Brenta ${ }^{2}$ \\ ${ }^{1}$ Evgenidion Hospital, Unit of Endocrinology Diabetes and Metabolism, Thyroid Section, University of Athens, Greece; \\ ${ }^{2}$ Dr. Cesar Milstein Hospital, Buenos Aires, Argentina
}

\begin{abstract}
L-thyroxine (LT4) treatment of hypothyroidism, particularly in patients with thyroid- stimulating hormone $(\mathrm{TSH})>10 \mathrm{mU} / \mathrm{L}$, results in improved lipid profile, as LT4 stimulates low-density lipoprotein cholesterol (LDL-C) degradation and the conversion of cholesterol in bile acids by inducing LDL-receptor and 7 alpha hydroxylase expression, respectively. Statins decrease total cholesterol (TC) and LDL-C mainly by suppressing 3-hydroxy-3-methylglutaryl coenzyme A activity. Therefore, the addition of statins to LT4 treatment, following the reversal of hypothyroidism, acts synergistically and forms a powerful treatment modality in patients with this condition whose serum lipids have not achieved the target. Statin add-on therapies, such as ezetimibe (EZE) and the recently approved proprotein convertase subtilisin/kexin type 9 (PCSK9) inhibitors, in conjunction with LT4 therapy, produce an even more favorable profile. Ezetimibe blocks the intestinal absorption of cholesterol, while in patients with heterozygous familiar hyperlipidemia, PCSK9 inhibitors suppress PCSK9, thereby promoting LDL-C degradation. The synergy of these drugs results in a far better lipidemic profile than when each drug is administered alone, with LT4 treatment clearly enhancing, in many respects, the efficacy of therapy.
\end{abstract}

Key words: Dyslipidemia, Ezetimibe, Familial hyperlipidemia, Hypothyroidism, Lipids, PCSK9, Statins, Thyroxine

\section{INTRODUCTION}

Thyroid hormones are known to be the main regulators of total cholesterol (TC) and lipoproteincholesterol metabolism. ${ }^{1}$ In this context, the impact of hypothyroidism, often linked to excess TC and

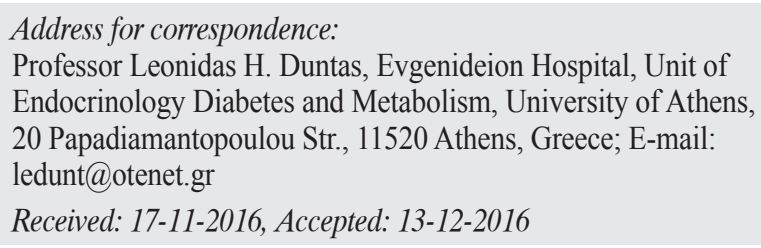

Received: 17-11-2016, Accepted: 13-12-2016

low-density lipoprotein cholesterol (LDL-C), on the arterial vessels was reported about 50 years ago when Steinberg, in an autopsy study, identified the narrowing of coronary arteries in myxedema and hypertensive patients. ${ }^{2}$ Some years later, Bastenie demonstrated that asymptomatic thyroid autoimmune disease, independently of other known risk factors, may be a predictor of subsequent development of coronary heart disease (CHD), the risk increasing with age. ${ }^{3}$ Though cardiovascular disease (CVD) has remained for several decades a leading cause of death worldwide, 
a reduction in TC from 1980 to 2000 brought about a $33 \%$ decrease in CHD deaths in the United States and a 19\%-46\% decrease in other developed countries. ${ }^{4}$ In the meantime, LDL-C has widely substituted TC as a risk biomarker and now forms the primary treatment target for hyperlipidemia. In the framework of this endeavor, it has been shown that a decline in LDL-C levels by means of statin-based therapies leads in a constant and graded manner to a decline in the risk of nonfatal $\mathrm{CV}$ events and mortality. ${ }^{4} \mathrm{~A}$ long-term evaluation of three studies disclosed that men with serum TC levels of $240 \mathrm{mg} / \mathrm{dL}$ or greater $(\geq 6.21$ $\mathrm{mmol} / \mathrm{L}$ ) had a 1-1.5 higher risk of CHD mortality compared to those with TC levels below $200 \mathrm{mg} / \mathrm{dL}$ $[<5.17 \mathrm{mmol} / \mathrm{L}]$ ), while the CVD disease mortality risk was 2.10 to 2.87 times greater. ${ }^{5}$ It is of note that in patients $<50$ years of age, TC levels were directly related to CVD mortality by $9 \%$ for each $10 \mathrm{mg} / \mathrm{dL}$ $\mathrm{TC}$, this clearly demonstrating that low TC improves longevity. ${ }^{6}$ In contrast, after the age 50 years there was no increased overall mortality with either high or low serum TC levels, probably due to the presence of comorbidities influencing mortality. In this line of evidence, a trajectory analysis using a mixed modeling approach in the Framingham Offspring Study revealed an independent association between early adult exposures to elevated LDL-C and diastolic blood pressure (DBP) and CHD events later in life. ${ }^{7}$ Hence, it is recommended that LDL-cholesterol be reduced in accordance with each patient's risk profile. ${ }^{\text {? }}$

Statins are considered the standard of care, since for more than three decades they have proven to be relatively safe and reliable agents in reducing $\mathrm{TC}$ and LDL-C, while they retard progression and even induce regression of mild noncalcified coronary plaque. ${ }^{8,9} \mathrm{In}$ cases in which LDL-C should be further decreased, other compounds, such as ezetimibe (EZE), can be added..$^{10}$ Nevertheless, in patients with very high LDLC, i.e. above $200 \mathrm{mg} / \mathrm{dl}$, as, for example, those with familiar hypercholesterolemia, the target is often not met. The recently introduced proprotein convertase subtilisin/kexin type 9 (PCSK9) inhibitors are indicated primarily for heterozygous familiar hyperlipidemia $(\mathrm{HeFH})$ patients, statin-intolerant patients and those at high CVD risk who are not able to reach the LDL-C target level with a high-potency statin dose. ${ }^{11}$

It is notable that the American College of Cardi- ology (ACC)/American Heart Association (AHA) (ACC/AHA) 2013 guidelines favor a statin-oriented approach based on randomized trials alone. ${ }^{12}$ However, while the recommendation of specific statin regimens usually results in a substantial reduction in LDL-C, it may negatively impact patient engagement and adherence to treatment, consequently leading to the under-treatment of high-risk patients. ${ }^{13}$ Indeed, a recent study aiming to determine and compare the extent of lipid-lowering treatment with regard to whether to pursue LDL-C treatment targets has reported that in spite of statin therapy, LDL-C values were high, substantially missing the targer, particularly in highrisk patients. ${ }^{14}$ These results point to the need for the optimization of existing treatment strategies and an interactive effort to improve therapeutic management in clinical practice.

In this connection, it should be remembered that endocrine diseases, the commonest being hypothyroidism and type 2 diabetes mellitus, may induce or exacerbate existing dyslipidemia and impede achievement of optimal results through hypolipidemic drugs, bearing in mind that the prevalence of hypothyroidism is $4.3 \%$ in patients with hypercholesterolemia. ${ }^{15}$ Therefore, patients with dyslipidemia should be regularly checked for thyroid dysfunction, while cholesterol and its subfractions should be monitored in patients with thyroid failure.

This review aims to assess the impact of thyroid hormones (TH) on lipids, particularly in manifested and subclinical thyroid failure. In parallel, it will emphasize the diverse mechanisms of action and the significance of the synergistic effect of thyroxine treatment in conjunction with statins and new hypolipidemic compounds in patients with hypothyroidism and dyslipidemia.

\section{THYROID HORMONES AND LIPIDS IN MANIFESTED HYPOTHYROIDISM}

Manifested hypothyroidism is marked by increased hypercholesterolemia, and especially of LDL-C and apolipoprotein B (apo B). ${ }^{16,17}$ This is due a) to decreased fractional clearance of LDL by the reduced number of LDL receptors (LDL-R) in the liver and b) to diminishing control by T3 over sterol regulatory element-binding protein 2 (SREBP-2), which is crucial 
for the expression of the LDL-R. ${ }^{18}$ Interestingly, there are the same alterations of lipoprotein in hypothyroidism as in homozygous familial hypercholesterolemia (HFH), thus indicating the existence of an intrinsic and almost total defect of receptor-mediated LDL catabolism in HFH similar to the analogous but reversible abnormality in hypothyroidism. ${ }^{1}$

The action of TH on bile acids (BA) has recently emerged as a discernible hypocholesterolemic effect. Elevated levels of bile acids cause depletion of the hepatic cholesterol pool followed by an increase in the synthesis of cholesterol in the liver and the hepatic uptake of cholesterol from the circulation. In fact, it has been reported that $\mathrm{TH}$ can promote BA synthesis by stimulating the rate-limiting enzyme cholesterol $7 \alpha$-hydroxylase (CYP7A1), a key element in cholesterol catabolism, as shown in mice ${ }^{19}$ and lately in humans, ${ }^{20}$ thus lowering plasma cholesterol levels. Data concerning hypothyroidism and BA are scarce; however, it has recently been reported that thyroid-stimulating hormone (TSH) per se may directly diminish liver BA synthesis via the SREBP-2/HNF-4 $\alpha$ (hepatocyte nuclear factor 4)/CYP7A1) pathway in vivo and in vitro. ${ }^{21}$ Since TSH is increased in all forms of primary hypothyroidism, a direct negative action of TSH in the liver may be an alternative explanation for the detrimental effect of the lack of TH in lipid metabolism.

High-density lipoprotein (HDL) levels are normal or even elevated in severe hypothyroidism because of reduced activity of cholesteryl-ester transfer protein (CETP) and hepatic lipase (HL), which are both enzymes regulated by $\mathrm{TH}^{22,23}$ Apart from its role in HDL regulation, HL is involved in the conversion of intermediate density lipoproteins (IDL) to LDL. ${ }^{24}$ Moreover, its diminished activity in hypothyroidism has been associated with the accumulation of remnant-like particles (RLP) in the serum of hypothyroid patients. ${ }^{25}$ Remnant cholesterol is a contributor to residual cardiovascular risk and represents the cholesterol content of a subset of triglyceride-rich lipoproteins that include chylomicron remnants, very low-density lipoprotein (VLDL) and intermediate density lipoprotein (IDL) in the nonfasting state, and VLDL and IDL in the fasting state. ${ }^{26}$ Therefore, the accumulation of triglyceride-rich lipoproteins in hypothyroid patients may be considered as part of the atherogenic scenario in these patients. With regard to triglyceride levels in a hypothyroid condition, they tend to be high, mainly due to reduced activity of the enzymes involved as lipoprotein lipase (LPL) ${ }^{27}$ and $\mathrm{HL}^{23}$ and/or on account of enhanced triglyceride hepatic synthesis. ${ }^{28}$

A well known study from the Mayo $\mathrm{Clinic}^{29}$ revealed that of 295 hypothyroid patients $56 \%$ had Frederickson type IIa dyslipidemia (hypercholesterolemia), 34\% type IIb (hypercholesterolemia + hypertriglyceridemia) and $1.5 \%$ type IV (hypertriglyceridemia), while only 8.5 had no lipid alterations. These abnormalities of lipoprotein metabolism that present in almost $90 \%$ of hypothyroid patients underscore once more the need to rule out the possible presence of hypothyroidism in every dyslipidemic patient. ${ }^{30}$

\section{THYROID HORMONE AND LIPIDS IN SUBCLINICAL HYPOTHYROIDISM}

Subclinical hypothyroidism ( $\mathrm{SCH}$ ) is defined biochemically by elevated levels of TSH while TH levels are still within the normal range. Its prevalence has been reported in about $6 \%$ of the general population, although it may be $20 \%$ and above in women over 60 years old. Since SCH has been linked to cardiovascular risk, all the most recent authoritative Society Guidelines advocate aggressive case finding in subjects with any kind of cardiovascular risk, including dyslipidemia. ${ }^{30-32}$

It is noteworthy that in $\mathrm{SCH}$ and hypercholesterolemic patients, serum TSH and BA levels were significantly and negatively correlated. ${ }^{33}$ Each 1 $\mu \mathrm{IU} / \mathrm{mL}$ increase in TSH level was a decrease in log-transformed values of total BAs by 0.182 , after adjustment for confounding factors, including $\mathrm{TH}$, this suggesting that TSH is correlated with the total BA level independently of thyroid hormone. Interestingly, the association between TSH and serum total BAs was stronger in subjects younger than 65 years. ${ }^{34}$

The quantitative lipid changes described in $\mathrm{SCH}$ have recently been evaluated in a meta-analysis that included 16 observational studies. It was shown that serum TC, LDL-C and triglyceride levels were significantly elevated in patients with $\mathrm{SCH}$ compared with euthyroid individuals,${ }^{35}$ the weighted mean differences being $12.17 \mathrm{mg} / \mathrm{dl}, 7.01 \mathrm{mg} / \mathrm{dl}$ and 13.19 $\mathrm{mg} / \mathrm{dl}$, respectively $(\mathrm{P}<0.001$ for all). 
A prospective cohort study designed to determine the relationship between baseline thyroid status and incident atrial fibrillation, cardiovascular disease and mortality in older men and women not taking thyroid medication recruited a total of 3,233 US communitydwelling individuals aged 65 years or older. ${ }^{36}$ While an association between subclinical hyperthyroidism and development of atrial fibrillation was observed, there was no finding of an association between subclinical hypothyroidism and cardiovascular disorders or mortality. ${ }^{36}$

Moreover, although levothyroxine (LT4) reverses the lipid alterations in manifested hypothyroidism, especially in patients with TSH above $10 \mathrm{mU} / \mathrm{L}, 1,37$ there is still controversy as to whether to prescribe treatment in $\mathrm{SCH}$ with this goal. It has also been demonstrated that treatment may improve carotid intima media thickness and other markers associated with CV disease, such as indices of endothelial function. ${ }^{37}$ However, in a recent evidence review of the U.S. Preventive Services Task Force, including MEDLINE and Cochrane databases through July 2014, it was observed that differences between treatment with levothyroxine and no treatment in $\mathrm{TC}$ and LDL-C ranged from -8 to $0 \mathrm{mg} / \mathrm{dL}$ and -22 to $2 \mathrm{mg} /$ dl, respectively. ${ }^{38}$

Though there is still a need for larger, randomized trials to elucidate this issue, a potential beneficial effect on lipid levels upon treatment can be inferred from this analysis.

Another important aspect is the quality of lipoproteins in $\mathrm{SCH}$ patients. As mentioned, impaired lipoprotein structure, such as in triglyceride-rich lipoproteins, has been associated with higher cardiovascular risk. These heterogeneous particles have reduced LDL-R affinity and accumulate in the subendothelial space where they are prone to oxidative modification and participate in the initiating event in atherogenesis. ${ }^{39}$ However, structural abnormalities of lipoproteins cannot be detected in routine blood tests and, unless specific measurements are obtained, they often go undiagnosed. In SCH there have been reports of augmented oxidation of LDL particles, ${ }^{40}$ increased postprandial lipemia ${ }^{41}$ impaired chemical composition with triglyceride enrichment of the LDL particle ${ }^{42}$ and qualitative evidence of RLP in the fasting serum of $\mathrm{SCH}$ women. ${ }^{43}$ As regards RLP quantification, particularly high levels have been reported both in Japanese ${ }^{44}$ and Argentinian ${ }^{45}$ cohorts of SCH patients. In addition, it was shown that the levels of these atherogenic particles decrease with levothyroxine, this probably related to increased degradation by $\mathrm{LH} .{ }^{46}$ Taken together, these findings suggest that $\mathrm{SCH}$ patients, even those with a normal lipid profile, are exposed to an elevated atherogenic risk due to the presence of LDL particles of altered composition, together with an accumulation of remnant lipoproteins that may be attributable to reduced HL activity. However, all these changes are reversible upon levothyroxine treatment.

\section{COMBINATION THERAPY}

\section{Thyroid hormones and statins}

It is well known that high levels of LDL-C, as well as of its structural protein, apo B 100, are directly associated with risk for atherosclerotic cardiovascular events, ${ }^{39}$ infiltration and retention of apo B-100 containing lipoproteins in the artery wall being a determinant event that ignites inflammation and promotes the development of atherosclerosis.

3-hydroxy-3-methylglutaryl coenzyme A (HMG$\mathrm{CoA}$ ) reductase inhibitors (statins) significantly lower LDL-C levels, resulting in a reduction in the risk of nonfatal $\mathrm{CV}$ events mortality in a continuous and graded manner over a wide range of baseline risk factors and LDL-C values. ${ }^{4}$ In hypothyroid patients treatment with LT4 usually decreases both TC and LDL-C $;{ }^{47}$ however, the effect of LT4 treatment on lipids, despite implementation of lifestyle modifications, is often not enough to achieve the therapeutic target, this dictating the use of statins. Of particular note in this respect is the fact that the addition of statins to LT4 therapy results in a greater reduction of $\mathrm{TC}$ and LDL-C than when each drug is administered singly. The mechanism is synergistic, LT4 primarily acting by inducing LDL-R expression and accelerating the degradation of LDL-C, and secondarily by inducing Cyp7a1 expression that stimulates the conversion and excretion of cholesterol as bile acids. Meanwhile, statins block the synthesis of cholesterol in the liver by inhibiting HMG-CoA reductase activity $^{48}$ (Figure 1). 
A recent retrospective population study, including 10,999 patients, was designed to determine the extent of drug interactions affecting LT4 intake over a follow-up of 20 years. It was shown that the administration of statins to the patients on long-term levothyroxine therapy resulted in a decrease of TSH by $0.17 \mathrm{mU} / 1$ concentration, while TSH diminished by $5 \mathrm{mU} / 1$ in $3 \cdot 7 \%$ of the study patients. ${ }^{49}$ Although these results await further confirmation, they may indicate a significant interaction of statins with LT4. On the other hand, other studies failed to find any interaction between statins and LT4, although these were smaller studies and of a much shorter followup period. ${ }^{50,51}$

Statins are likely to have an immune-modulatory role in thyroid autoimmunity, as in vivo and in vitro studies showed an increase in serum FT3, FT4, and concomitantly a reduction in TSH levels, while CD4+ cells and B lymphocytes rose and CD8+ cells, natural killer cells and activated $T$ lymphocytes significantly diminished. ${ }^{52}$ According to the authors, these results are mediated by activation of apoptosis by the statins. These findings were indirectly corroborated by another study using high-dose statin therapy and reporting a significant reduction of thyroid peroxidase antibodies (TPOAB) and a decline in thyroglobulin antibodies $(\operatorname{Tg} \mathrm{AB})$ in women with Hashimoto thyroiditis. ${ }^{53}$ However, this was reversed when the statin dose was reduced and EZE was added, indicating that statins have an effect on markers of autoimmunity when administered at a high dose. This can probably be related to the reduction of inflammation following the decrease of cholesterol production. In this line of evidence, it was recently reported that statins significantly lower systemic inflammation as measured by C-reactive protein,${ }^{54}$ also that statins are protective against inflammatory gastrointestinal diseases

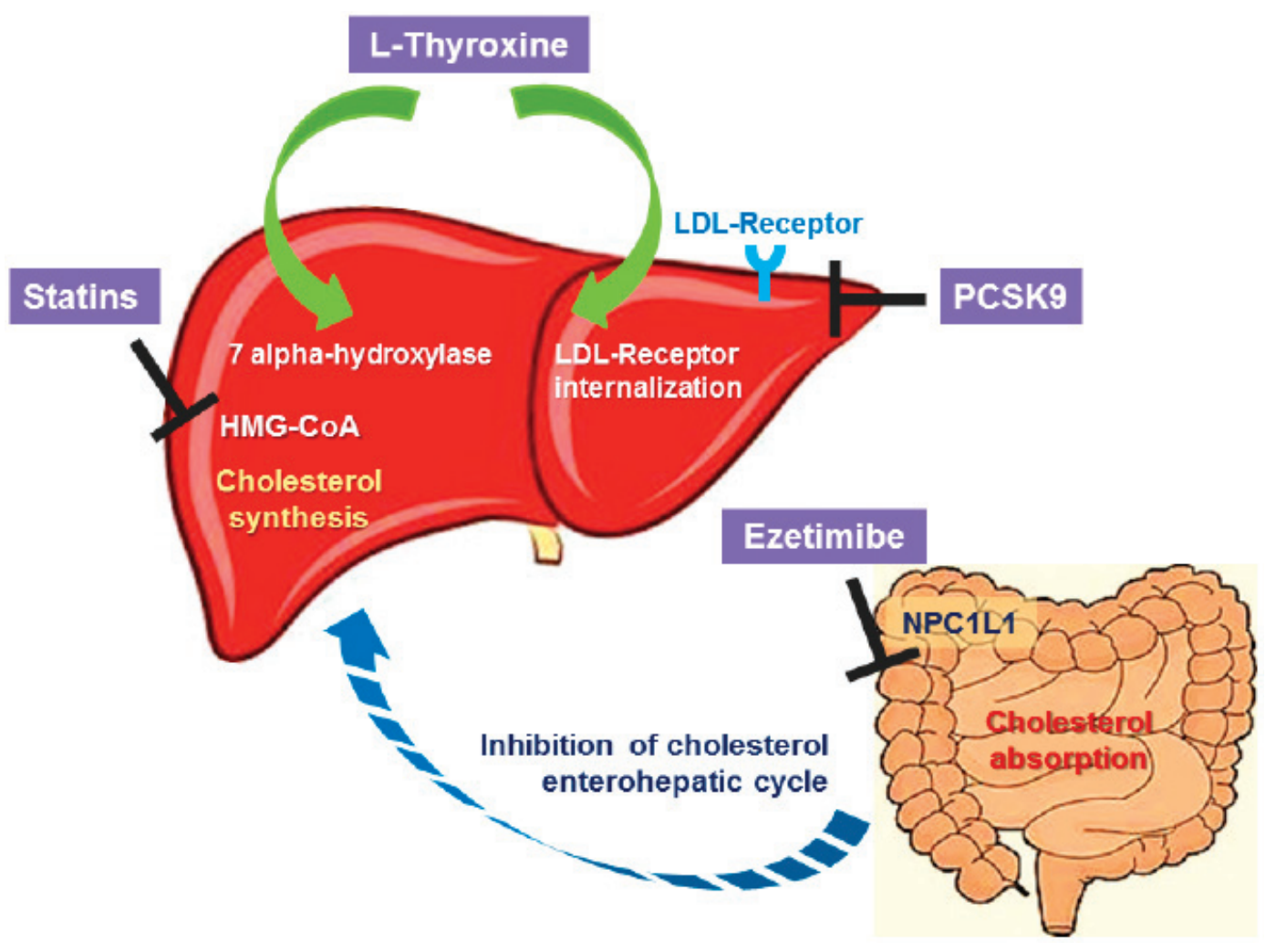

Figure 1. By enhancing 7 alpha hydroxylase and LDL-receptor expression at the hepatocyte, thyroid hormone regulates the catabolism of cholesterol. Statins inhibit the synthesis of cholesterol by suppressing the activity of 3-hydroxy-3-methylglutaryl coenzyme A reductase (HMG-CoA), while ezetimibe, by inhibiting the Niemann-Pick C1-Like 1 (NPC1L1) sterol receptor in intestinal enterocytes and within the liver, significantly blocks exogenous cholesterol absorption. Proprotein convertase subtilisin/kexin type 9 (PCSK9) blockers inhibit the internalization of the LDL-receptor and promote LDL-C degradation. 
like Crohn's disease and ulcerative colitis. Also of interest is a recent retrospective study evaluating 135 dyslipidemic patients receiving statin therapy for at least 5 years who were compared with 137 controls. Those treated with statins showed a remarkably lower prevalence of thyroid nodules (36.3\% vs. $67.9 \%$, P $<0.001)$ and a reduced number and smaller total volume of lesions as compared with the control group. ${ }^{55}$ According to the authors, an anti-proliferative and/or pro-apoptotic effect of long-term statin treatment on thyroid cells might be an explanation. ${ }^{55}$

However, caution is required in treating manifested and subclinical hypothyroid patients with statins, as hypothyroid patients are more likely to have adverse muscle effects and increased serum levels of creatine kinase (CK). ${ }^{56}$ Muscle syndromes are, in fact, the most common side effects following initiation of statin therapy, being observed in about $10-15 \%$ of patients. ${ }^{57}$ Accordingly, since the administration of statins before the achievement of euthyroidism may exacerbate myalgias or else cause rhabdomyoysis marked by extreme levels of $\mathrm{CK}$, it is crucial that treatment with statins be preceded by the chemical reversal of hypothyroidism. In this connection, the recent statement of the European Atherosclerosis Society (EAS) has introduced the term statin-associated muscle symptoms (SAMS), replacing the term 'statin intolerance'. ${ }^{58}$

Moreover, in patients with thyroid cancer in preparation for radioiodine scan and treatment, withdrawal of LT4 should be exercised with extreme care and close monitoring, since severe myopathy in such patients has been reported..$^{59}$ If indicated, recombinant human TSH injections should be preferred to levothyroxine withdrawal in the latter cases.

Another effect of statins is inhibition of Sec-tRNA isopentenylation, a side road of the mevalonate pathway. ${ }^{59}$ Sec-tRNA isopentenylation functionally requires isopentenyl pyrophosphate, which forms the core of cholesterol building and whose suppression may result in a decrease of selenoprotein expression: this possibly explains some of the pleiotropic effects of statins, such as immunosuppressive and redox activity. ${ }^{59}$ Since chronic administration of statins is therefore likely to seriously impact the function of selenium-dependent selenoproteins, periodic meas- urement of serum selenium or selenoprotein $\mathrm{P}$, the storage protein of selenium, is advisable.

\section{Thyroid hormones and ezetimibe}

Despite a $25-30 \%$ reduction in CVD risk with LDL-C lowering strategies, there is still a significant residual risk, and in such cases where the LDL-C target is not achieved with statin treatment it has been proposed that EZE should be added. ${ }^{60}$ Adding EZE to statins monotherapy offers a reasonable and safe treatment option to enable patients at persistent risk of CHD to achieve the aggressive LDL-C-lowering goals recommended by recent treatment guidelines. EZE binds to an extracellular loop of the NiemannPick C1-Like 1 (NPC1L1) sterol receptor on intestinal enterocytes and inhibits its sterol transport function. ${ }^{61}$ NPC1L1 localizes to the brush border membrane of absorptive enterocytes in the small intestine and its intestinal expression is downregulated by nutritive high cholesterol levels. ${ }^{61}$ Thus, by inhibiting the NPC1L1 sterol receptor in intestinal enterocytes and within the liver, EZE selectively blocks exogenous cholesterol absorption and decreases intestinal delivery of cholesterol to the liver. Furthermore, the addition of the latter drug has been recorded as substantially augmenting the rate of ESC/EAS LDL-C target achievement, thus further decreasing LDL-C by $15 \%$ to $20 \%$ while also increasing the rate of the LDL-C lowering response proposed by the ACC/AHA. ${ }^{12,13}$ In the IMPROVE-IT study, the largest and longest study of lipid-lowering drugs including 18,000 high-risk CV patients, the addition of EZE to simvastatin versus simvastatin monotherapy significantly reduced the primary $\mathrm{CV}$ endpoint from $34.7 \%$ to $32.7 \%$ within 7 years. ${ }^{62}$ The combination of a statin with EZE represents an attractive strategy for cholesterol-lowering treatment, as both sources of cholesterol are inhibited: a) endogenous biosynthesis (inhibited by statins), b) absorption from the intestine (inhibited by EZE).

A recent 6-week, double-blind, randomized, parallel group study of 1,128 subjects with hypercholesterolemia evaluated the effects of metabolic syndrome (MetS) factors and insulin resistance on EZE/simvastatin and/or atorvastatin treatment efficacy in patients with MetS. ${ }^{63}$ It is worth mentioning that while the magnitude of lipid-altering effects produced by each treatment regimen was relatively similar, the 
EZE/simvastatin group exhibited a greater percent reduction in lipid fractions than atorvastatin alone.

It is of particular interest that in patients under treatment with LT4, the co-administration of EZE does not significantly affect LT4 absorption, ${ }^{64}$ EZE being expressed in macrophages at a much lower rate than in enterocytes. ${ }^{55,65}$ In human macrophages, EZE, by binding to specific cell surface receptors, may exert pleiotropic effects by decreasing the number of monocytes/macrophages in atherosclerotic lesions. ${ }^{65}$ EZE can also reduce the lymphocyte secretory function, while it enhances the statin suppressive effect on lymphocyte cytokine release. ${ }^{65,66}$ It is well known that monocytes/macrophages and lymphocytes play an important role in the progression of autoimmunity, thus EZE may theoretically alleviate the inflammatory process in patients with autoimmune disorders, particularly when administered with statins which possess immunosuppressive properties ${ }^{67}$ (Figure 2). EZE has not been related to any muscle side effects, although information regarding hypothyroid patients under treatment with EZE or with PCSK9 is not at present available.

It is also worth noting that in hypophysectomized rats, the absorption of dietary cholesterol is under pituitary control exerted by TH. Secretion of cholesterol into bile is an important step for the elimination of cholesterol from the body via adenosine triphosphate (ATP)-binding cassette, subfamily G (WHITE), member 5 (ABCG5) and ABCG5/G8 transporters. ${ }^{68}$ The expression of both transporters is stimulated by $\mathrm{TH}$ treatment. ${ }^{69}$ Thus, hepatic ABCG5/G8 overexpression induced by TH may increase biliary excretion, resulting in reduction of atherogenic risk. On the other hand, it has been revealed that the expression of Niemann-Pick C2 (NPC2) - a cholesterol-binding protein secreted by the biliary system-positively regulates the biliary secretion of cholesterol. ${ }^{70}$

\section{Thyroid hormones and PCSK9}

Proprotein CSK9, which is secreted by the liver into the plasma, has been associated with higher

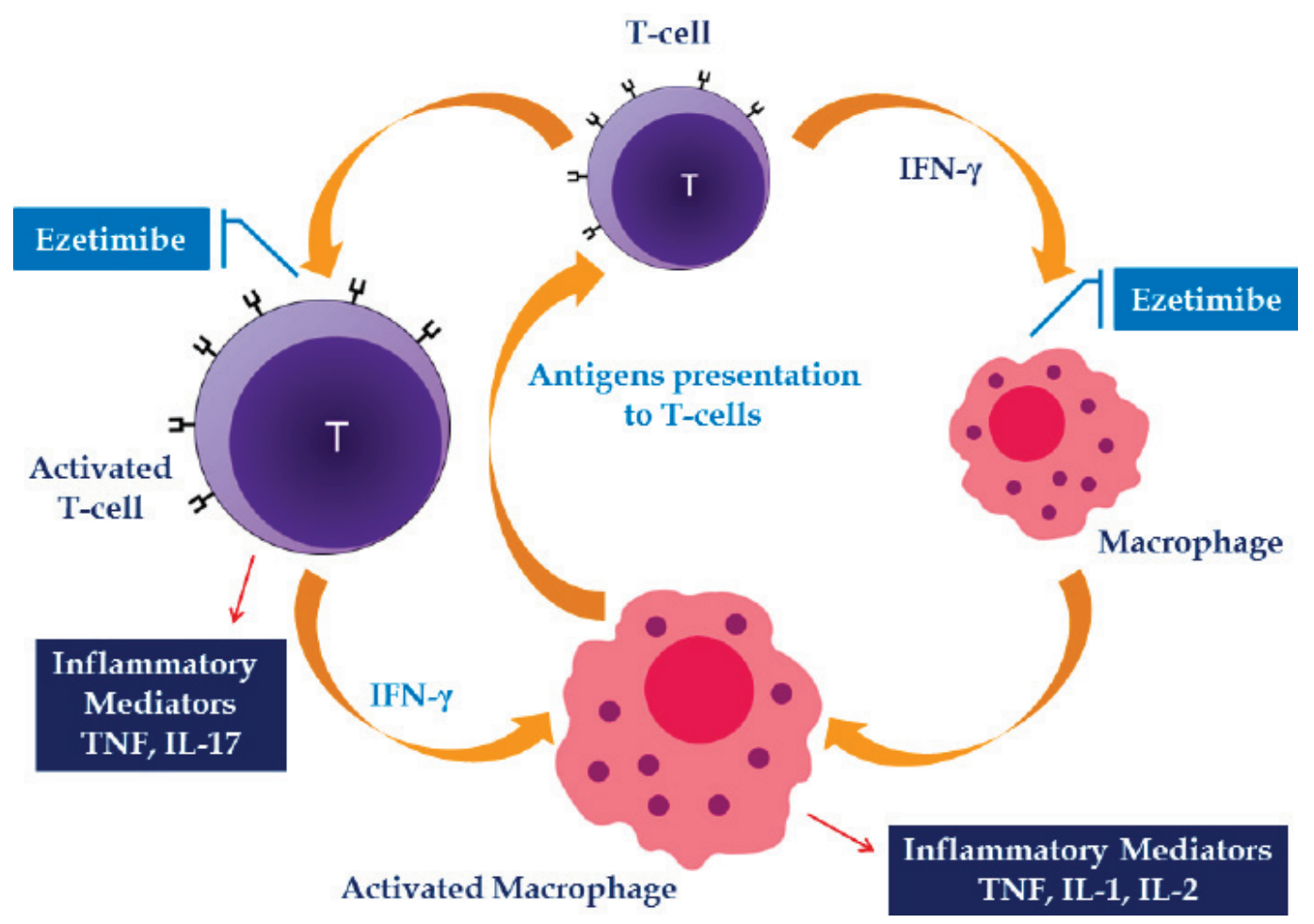

Figure 2. The immune-modulatory effects of ezetimibe (EZE). EZE is expressed in macrophages by binding to specific cell surface receptors, while it exerts pleiotropic effects by decreasing the number of monocytes/macrophages in atherosclerotic lesions and by reducing the secretory function of lymphocytes. It can also enhance the statin suppressive effect on lymphocyte cytokine release. Thus, EZE is likely to reduce the activation of macrophages by cytokines. 
$\mathrm{CV}$ risk and steatosis. ${ }^{71}$ It moreover binds to the LDL-R on the surface of hepatocytes, thereby preventing its recycling and enhancing its degradation into lysosomes, leading to reduced LDL-cholesterol clearance. ${ }^{71}$ The recent approval of the first two monoclonal antibodies, alirocumab and evolocumab which target PCSK9, provides an eclectic treatment for patients with familiar hyperlipidemia (FH) and/ or those with CVD who are statin-resistant. ${ }^{72-74}$ Both alirocumab and evolocumab brought about LDL-C reductions of a similar magnitude to those seen in non-FH subjects. FH is a dominantly inherited error of metabolism, a life-threatening disease marked by elevated plasma LDL-C and usually of very-lowdensity lipoprotein (VLDL) remnants, xanthomas of skin and tendons and premature heart disease due to deposition of lipids in xanthomas and the coronary arterial walI. ${ }^{74}$ Deficiency of LDL-R accounts for the increased plasma concentrations of LDL and VLDL remnants in $\mathrm{FH} .{ }^{75}$ Meanwhile, the fractional rates of catabolism of LDL and VLDL remnants are low, with the clinical and biochemical aspects being more prominent in homozygotes than in heterozygotes. In patients with $\mathrm{HeFH}$, moderate-to-high intensity statin therapy contributes to a lower risk of CVD and mortality by up to $44 \% .{ }^{76}$ Treatment with statins increases plasma PCSK9 levels in proportion to the magnitude of LDL-reduction, ${ }^{77}$ this most probably constituting a compensating mechanism for the increase in intrahepatic cholesterol induced by the drugs. It can therefore be speculated that patients with hypothyroidism under hypolipidemic treatment may obtain additional benefit from levothyroxine treatment through reduction of the levels of PCSK9. In a multinational, cross-sectional, observational study (DYSIS) of 54,811 adult outpatients receiving statin therapy, the percentages of patients with high levels of LDL-C, accompanied by probable FH, were assessed across 29 countries. ${ }^{78}$ Interestingly, despite intensive statin therapy, high LDL-C levels and higher prevalence of $\mathrm{FH}$ were observed in some countries and in younger age patients (45-54 years), with CHD being exhibited in $>60 \%$ of patients with probable $\mathrm{FH}^{79}$

The effects of TH on cholesterol include its action on suppressing PCSK9, the latter gene being under the regulatory control of SREBP2, which is regulated in turn by T3 ${ }^{80}$ Recently it was tested whether, and to what extent, plasma PCSK9 correlates with thyroid function in euthyroid subjects who were non-obese and in others who were obese to varying degrees ${ }^{81}$ PCSK9 correlated positively with TSH in the non-obese but not in the obese subjects. The effect of TSH on PCSK9 was blunted by increasing adiposity, supporting the hypothesis that variations in thyroid function, even within the euthyroid range, may influence lipoprotein cholesterol metabolism. ${ }^{82}$ The relation of TSH levels to lipoproteins and hypercholesterolemia is linear in euthyroid subjects, who display higher $\log \mathrm{TC}$ and log triglyceride levels characterized by increasing serum TSH levels, the effects of TSH on TC levels being both direct and indirect and acting probably via the PCSK9 pathway in non-obese subjects, a relationship that appears to be disturbed in obesity. Moreover, PCSK9 may have a role within the context of metabolic syndrome (MetS) and be associated with such parameters as lipids, glucose homeostasis and inflammation..$^{83}$

It should be stated that in hyperthyroid individuals, a concomitant reduction in LDL-C and PCSK9 levels has been reported,${ }^{84}$ strongly indicating the existence of alternative pathways, other than the transcriptional stimulation of the LDL-R gene, whereby TH regulates hepatic LDL-R

\section{CONCLUSIONS}

Given that thyroid hormone is the main regulator of lipid metabolism, in cases of hypothyroidism, and by extension of hyperlipidemia, cholesterol metabolism is deranged to a larger or smaller degree depending upon the disease type and its severity. While treatment with LT4 alone reverses secondary hyperlipidemia, when hyperlipidemia persists, as in cases of primary hyperlipidemia or $\mathrm{HeFH}$, administration of statins together with ezetimibe add-on and PCSK9 inhibitors should be considered. The combination of these drugs is likely to augment the efficacy of the therapy because of the multiple effects of LT4 at the molecular level, while its benefit at the individual level is that by permitting a potential reduction of statin dosage it improves patient acceptance and compliance. 


\section{REFERENCES}

1. Duntas LH, Brenta G, 2012 The effect of thyroid disorders on lipid levels and metabolism. Med Clin North Am 96: 269-281.

2. Steinberg AD, 1968 Myxedema and coronary artery disease--a comparative autopsy study. Ann Intern Med 68: 338-344.

3. Bastenie PA, Vanhaelst L, Golstein J, Smets P, 1977 Asymptomatic autoimmune thyroiditis and coronary heart-disease. Cross-sectional and prospective studies. Lancet 2: 155-158.

4. Wadhera RK, Steen DL, Khan I, Giugliano RP, Foody JM, 2016 A review of low-density lipoprotein cholesterol, treatment strategies, and its impact on cardiovascular disease morbidity and mortality. J Clin Lipidol 10: 472-489.

5. Stamler J, Daviglus ML, Garside DB, Dyer AR, Greenland P, Neaton JD, 2000 Relationship of baseline serum cholesterol levels in 3 large cohorts of younger men to long-term coronary, cardiovascular, and all-cause mortality and to longevity. JAMA 284: 311-318.

6. Anderson KM, Castelli WP, Levy D, 1987 Cholesterol and mortality. 30 years of follow-up from the Framingham study. JAMA 257: 2176-2180.

7. Pletcher MJ, Vittinghoff E, Thanataveerat A, BibbinsDomingo K, Moran AE, 2016 Young adult exposure to cardiovascular risk factors and risk of events later in life: The Framingham Offspring Study. PLoS One 11: e0154288.

8. März W, Scharnagl H, Gouni-Berthold I, et al, 2016 LDL-Cholesterol: Standards of treatment 2016: A German Perspective. Am J Cardiovasc Drugs 16: 323-336.

9. Li Z, Hou Z, Yin W, et al, 2016 Effects of statin therapy on progression of mild noncalcified coronary plaque assessed by serial coronary computed tomography angiography: A multicenter prospective study. Am Heart J 180: 29-38.

10. Gotto AM Jr, Farmer JA, 2006 Drug insight: the role of statins in combination with ezetimibe to lower LDL cholesterol. Nat Clin Pract Cardiovasc Med 3: 664-672.

11. Blom DJ, Dent R, Castro RC, Toth PP, 2016 PCSK9 inhibition in the management of hyperlipidemia: focus on evolocumab. Vasc Health Risk Manag 12: 185-197.

12. Ray KK, Kastelein JJ, Boekholdt SM, et al, 2014 The ACC/AHA 2013 guideline on the treatment of blood cholesterol to reduce atherosclerotic cardiovascular disease risk in adults: the good the bad and the uncertain: a comparison with ESC/EAS guidelines for the management of dyslipidaemias 2011. Eur Heart J 35: 960-968.

13. Barkas F, Milionis H, Kostapanos MS, Mikhailidis DP, Elisaf M, Liberopoulos E, 2015 How effective are the ESC/EAS and 2013 ACC/AHA guidelines in treating dyslipidemia? Lessons from a lipid clinic. Curr Med Res Opin 31: 221-228.

14. Gitt AK, Lautsch D, Ferrieres J, et al, 2016 Low-density lipoprotein cholesterol in a global cohort of 57,885 statin-treated patients. Atherosclerosis 255: 200-209.

15. Tagami T, Kimura H, Ohtani S, et al. PHPH study group, 2011 Multi-center study on the prevalence of hypothyroidism in patients with hypercholesterolemia. Endocr J 58: 449-457.

16. Baron DN, 1956 Hypothyroidism; its aetiology and relation to hypometabolism, hypercholesterolaemia, and increase in body-weight. Lancet 271: 277-281.

17. Duntas LH, 2002 Thyroid and lipids. Thyroid 12: 287293.

18. Shin DJ, Osborne TF, 2003 Thyroid hormone regulation and cholesterol metabolism are connected through sterol regulatory element-binding protein-2 (SREBP-2). J Biol Chem 278: 34114-34118.

19. Ness GC, Pendleton LC, Li YC, Chiang JY, 1990 Effect of thyroid hormone on hepatic cholesterol 7 alpha hydroxylase, LDL receptor, HMG-CoA reductase, farnesyl pyrophosphate synthetase and apolipoprotein A-I mRNA levels in hypophysectomized rats. Biochem Biophys Res Commun 172: 1150-1156.

20. Lammel Lindemann JA, Angajala A, Engler DA, Webb P, Ayers SD, 2014 Thyroid hormone induction of human cholesterol 7 alpha-hydroxylase (Cyp7a1) in vitro. Mol Cell Endocrinol 388: 32-40.

21. Song Y, Xu C, Shao S, et al, 2015 Thyroid-stimulating hormone regulates hepatic bile acid homeostasis via SREBP-2/HNF-4alpha/CYP7A1 axis. J Hepatol 62: 1171-1179.

22. Tan KC, Shiu SW, Kung AW, 1998 Effect of thyroid dysfunction on high-density lipoprotein subfraction metabolism: roles of hepatic lipase and cholesteryl ester transfer protein. J Clin Endocrinol Metab 83: 2921-2924.

23. Valdemarsson S, Nilsson-Ehle P, 1987 Hepatic lipase and the clearing reaction: studies in euthyroid and hypothyroid subjects. Horm Metab Res 19: 28-30.

24. Zambon A, Deeb SS, Bensadoun A, Foster KE, Brunzell JD, 2000 In vivo evidence of a role for hepatic lipase in human apoB-containing lipoprotein metabolism, independent of its lipolytic activity. J Lipid Res 41: 2094-2099.

25. Ito M, Takamatsu J, Matsuo T, et al, 2003 Serum concentrations of remnant-like particles in hypothyroid patients before and after thyroxine replacement. Clin Endocrinol (Oxf) 58: 621-626.

26. Varbo A, Benn M, Nordestgaard BG, 2014 Remnant cholesterol as a cause of ischemic heart disease: evidence, definition, measurement, atherogenicity, high risk patients, and present and future treatment. Pharmacol Ther 141: 358-367.

27. Kuusi T, Taskinen MR, Nikkila EA, 1988 Lipoproteins, lipolytic enzymes, and hormonal status in hypothyroid women at different levels of substitution. J Clin Endocrinol Metab 66: 51-56.

28. Gjedde S, Gormsen LC, Rungby J, et al, 2010 Decreased lipid intermediate levels and lipid oxidation rates de- 
spite normal lipolysis in patients with hypothyroidism. Thyroid 20: 843-849.

29. O’Brien T, Dinneen SF, O’Brien PC, Palumbo PJ, 1993 Hyperlipidemia in patients with primary and secondary hypothyroidism. Mayo Clin Proc 68: 860-866.

30. Brenta G, Vaisman M, Sgarbi JA, et al, 2013 Clinical practice guidelines for the management of hypothyroidism. Arq Bras Endocrinol Metabol 57: 265-291.

31. National Cholesterol Education Program (NCEP) Expert Panel on Detection, Evaluation, and Treatment of High Blood Cholesterol in Adults (Adult Treatment Panel III), 2002 Third Report of the National Cholesterol Education Program (NCEP) Expert Panel on Detection, Evaluation, and Treatment of High Blood Cholesterol in Adults (Adult Treatment Panel III) final report. Circulation 106: 3143-3421.

32. Garber JR, Cobin RH, Gharib H, et al, 20122012 Clinical practice guidelines for hypothyroidism in adults: cosponsored by the American Association of Clinical Endocrinologists and the American Thyroid Association. Endocr Pract 18: 988-1028.

33. Song Y, Zhao M, Zhang H, et al, 2016 Thyroid stimulating hormone levels are inversely associated with serum total bile acid levels: a cross-sectional study. Endocr Pract 22: 420-426.

34. Liu XL, He S, Zhang SF, et al, 2014 Alteration of lipid profile in subclinical hypothyroidism: a meta-analysis. Med Sci Monit 20: 1432-1441.

35. Danese MD, Ladenson PW, Meinert CL, Powe NR, 2000 Clinical review 115: effect of thyroxine therapy on serum lipoproteins in patients with mild thyroid failure: a quantitative review of the literature. J Clin Endocrinol Metab 85: 2993-3001.

36. Cappola AR, Fried LP, Arnold AM, et al, 2006 Thyroid status, cardiovascular risk, and mortality in older adults. JAMA 295: 1033-1041.

37. Lioudaki E, Mavroeidi NG, Mikhailidis DP, Ganotakis ES, 2013 Subclinical hypothyroidism and vascular risk: an update. Hormones (Athens) 12: 495-506.

38. Rugge JB, Bougatsos C, Chou R, 2015 Screening and treatment of thyroid dysfunction: an evidence review for the U.S. Preventive Services Task Force. Ann Intern Med 162: 35-45.

39. Linton MF, Yancey PG, Davies SS, et al In: De Groot LJ, Beck-Peccoz P, Chrousos G, et al (eds), 2000-2015 The Role of lipids and lipoproteins in atherosclerosis. SourceEndotext [Internet]. South Dartmouth (MA): MDText.com, Inc.

40. Duntas LH, Mantzou E, Koutras DA, 2002 Circulating levels of oxidized low-density lipoprotein in overt and mild hypothyroidism. Thyroid 12: 1003-1007.

41. Arikan S, Bahceci M, Tuzcu A, Celik F, Gokalp D, 2012 Postprandial hyperlipidemia in overt and subclinical hypothyroidism. Eur J Intern Med 23: e141-145.

42. Boren J, Williams KJ, 2016 The central role of arterial retention of cholesterol-rich apolipoprotein-B-containing lipoproteins in the pathogenesis of atherosclerosis: a triumph of simplicity. Curr Opin Lipidol 27: 473-483.

43. Brenta G, Berg G, Arias P, et al, 2007 Lipoprotein alterations, hepatic lipase activity, and insulin sensitivity in subclinical hypothyroidism: response to L-T(4) treatment. Thyroid 17: 453-460.

44. Ito M, Takamatsu J, Sasaki I, et al, 2004 Disturbed metabolism of remnant lipoproteins in patients with subclinical hypothyroidism. Am J Med 117: 696-699.

45. Brenta G, Berg G, Zago V, et al, 2008 Proatherogenic mechanisms in subclinical hypothyroidism: hepatic lipase activity in relation to the VLDL remnant IDL. Thyroid 18: 1233-1236.

46. Brenta G, Berg G, Miksztowicz V, et al, 2016 Atherogenic lipoproteins in subclinical hypothyroidism and their relationship with hepatic lipase activity: response to replacement treatment with levothyroxine. Thyroid 26: 365-372.

47. Lopez D, Abisambra Socarrás JF, Bedi M, Ness GC, 2007 Activation of the hepatic LDL receptor promoter by thyroid hormone. Biochim Biophys Acta 1771: 1216-1225.

48. Lin JZ, Martagón AJ, Hsueh WA, et al, 2012 Thyroid hormone receptor agonists reduce serum cholesterol independent of the LDL receptor. Endocrinology 153: 6136-6144.

49. Irving SA, Vadiveloo T, Leese GP, 2015 Drugs that interact with levothyroxine: an observational study from the Thyroid Epidemiology, Audit and Research Study (TEARS). Clin Endocrinol (Oxf) 82: 136-141.

50. Abbasinazari M, Nakhjavani M, Gogani S, 2011 The effects of simvastatin on the serum concentrations of thyroid stimulating hormone and free thyroxine in hypothyroid patients treated with levothyroxine. Iran J Med Sci 36: 80-83.

51. Rennert G, Rennert HS, Pinchev M, Gruber SB, 2010 A case-control study of levothyroxine and the risk of colorectal cancer. J Natl Cancer Inst 102: 568-572.

52. Gullu S, Emral R, Bastemir M, Parkes AB, Lazarus $\mathrm{JH}, 2005$ In vivo and in vitro effects of statins on lymphocytes in patients with Hashimoto's thyroiditis. Eur J Endocrinol 153: 41-48.

53. Krysiak R, Szkróbka W, Okopień B, 2016 The Effect of Ezetimibe/Statin combination and high-dose statin therapy on thyroid autoimmunity in women with Hashimoto's thyroiditis and cardiovascular disease: A pilot Study. Exp Clin Endocrinol Diabetes 124: 577-581.

54. Khalili H, 2016 Statins for Inflammatory Bowel Disease: Expanding the Scope of Prevention. Am J Gastroenterol 111: 1424-1426.

55. Cappelli C, Castellano M, Pirola I, et al, 2008 Reduced thyroid volume and nodularity in dyslipidaemic patients on statin treatment. Clin Endocrinol (Oxf) 68: 16-21.

56. Tokinaga K, Oeda T, Suzuki Y, Matsushima Y, 2006 HMG-CoA reductase inhibitors (statins) might cause high elevations of creatine phosphokinase $(\mathrm{CK})$ in patients 
with unnoticed hypothyroidism. Endocr J 53: 401-405.

57. Lando HM, Burman KD, 2008 Two cases of statininduced myopathy caused by induced hypothyroidism. Endocr Pract 4: 726-731.

58. Banach M, Rizzo M, Toth PP, et al, 2015 Statin intolerance - an attempt at a unified definition. Position paper from an International Lipid Expert Panel. Arch Med Sci 11: 1-23.

59. Moosmann B, Behl C, 2004 Selenoproteins, cholesterollowering drugs, and the consequences: revisiting of the mevalonate pathway. Trends Cardiovasc Med 14: 273-281.

60. Joy TR, 2012 Novel therapeutic agents for lowering low density lipoprotein cholesterol. Pharmacol Ther 135: 31-43.

61. Davis HR Jr, Altmann SW, 2009 Niemann-Pick C1 Like 1 (NPC1L1) an intestinal sterol transporter. Biochim Biophys Acta 1791: 679-683.

62. Cannon CP, Blazing MA, Giugliano RP, et al, 2015 Ezetimibe added to statin therapy after acute coronary syndromes. N Engl J Med 372: 2387-2397.

63. Rosen JB, Ballantyne CM, Hsueh WA, et al, 2015 Influence of metabolic syndrome factors and insulin resistance on the efficacy of ezetimibe/simvastatin and atorvastatin in patients with metabolic syndrome and atherosclerotic coronary heart disease risk. Lipids Health Dis 14: 103.

64. Ananthakrishnan S, Braverman LE, Levin RM, Magnani B, Pearce EN, 2008 The effect of famotidine, esomeprazole, and ezetimibe on levothyroxine absorption. Thyroid 18: 493-498.

65. Seedorf U, Engel T, Lueken A, et al, 2004 Cholesterol absorption inhibitor Ezetimibe blocks uptake of oxidized LDL in human macrophages. Biochem Biophys Res Commun 320: 1337-1341.

66. Krysiak R, Zmuda W, Okopien B, 2012 The effect of ezetimibe, administered alone or in combination with simvastatin, on lymphocyte cytokine release in patients with elevated cholesterol levels. J Intern Med 271: 32-42.

67. Moutzouri E, Tellis CC, Rousouli K, et al, 2012 Effect of simvastatin or its combination with ezetimibe on Toll-like receptor expression and lipopolysaccharide - induced cytokine production in monocytes of hypercholesterolemic patients. Atherosclerosis 225: 381-387.

68. Bonde Y, Plösch T, Kuipers F, Angelin B, Rudling M, 2012 Stimulation of murine biliary cholesterol secretion by thyroid hormone is dependent on a functional ABCG5/G8 complex. Hepatology 56: 1828-1837.

69. Gälman C, Bonde Y, Matasconi M, Angelin B, Rudling M, 2008 Dramatically increased intestinal absorption of cholesterol following hypophysectomy is normalized by thyroid hormone. Gastroenterology 134: 1127-1136.

70. Yamanashi Y, Takada T, Yoshikado T, Shoda J, Suzuki H, 2011 NPC2 regulates biliary cholesterol secretion via stimulation of ABCG5/G8-mediated cholesterol transport. Gastroenterology 140: 1664-1674.

71. Seidah NG, Awan Z, Chrétien M, Mbikay M, 2014 PCSK9: a key modulator of cardiovascular health. Circ Res. 114: 1022-1036.

72. Achimastos A, Alexandrides T, Alexopoulos D, et al, 2016 Expert consensus on the rational clinical use of proprotein convertase subtilisin/kexin type 9 (PCSK9) inhibitors. Hormones (Athens) 15: 8-14.

73. Saxon DR, Eckel RH, 2016 Statin Intolerance: A literature review and management strategies. Prog Cardiovasc Dis 59: 153-164.

74. Sullivan D, Olsson AG, Scott R, et al, 2012 Effect of a monoclonal antibody to PCSK9 on low-density lipoprotein cholesterol levels in statin-intolerant patients: the GAUSS randomized trial. JAMA 308: 2497-2506.

75. Myant NB, 1983 The metabolic basis of familial hypercholesterolemia. Klin Wochenschr 61: 383-401.

76. Thompson GR, Soutar AK, Spengel FA, et al, 1981 Defects of receptor-mediated low density lipoprotein catabolism in homozygous familial hypercholesterolemia and hypothyroidism in vivo. Proc Natl Acad Sci USA 78: 2591-2595.

77. Besseling J, Hovingh GK, Huijgen R, Kastelein JJ, Hutten BA, 2016 Statins in familial hypercholesterolemia: Consequences for coronary artery disease and all-cause mortality. Am Coll Cardiol 68: 252-260.

78. Awan Z, Seidah NG, MacFadyen JG, et al, 2012 Rosuvastatin, proprotein convertase subtilisin/kexin type 9 concentrations, and LDL cholesterol response: the JUPITER trial. Clin Chem 58: 183-189.

79. Catapano AL, Lautsch D, Tokgözoglu L, et al, 2016 Prevalence of potential familial hypercholesteremia (FH) in 54,811 statin-treated patients in clinical practice. Atherosclerosis 252: 1-8.

80. Dong B, Singh AB, Fung C, Kan K, Liu J, 2014 CETP inhibitors downregulate hepatic LDL receptor and PCSK9 expression in vitro and in vivo through a SREBP2 dependent mechanism. Atherosclerosis 235: 449-462.

81. Kwakernaak AJ, Lambert G, Muller Kobold AC, Dullaart RP, 2013 Adiposity blunts the positive relationship of thyrotropin with proprotein convertase subtilisin-kexin type 9 levels in euthyroid subjects. Thyroid 23: 166-172.

82. Wang F, Tan Y, Wang C, et al, 2012 Thyroid-stimulating hormone levels within the reference range are associated with serum lipid profiles independent of thyroid hormones. J Clin Endocrinol Metab. 97: 2724-2731.

83. Ferri N, Ruscica M, 2016 Proprotein convertase subtilisin/kexin type 9 (PCSK9) and metabolic syndrome: insights on insulin resistance, inflammation, and atherogenic dyslipidemia. Endocrine 54: 588-601.

84. Bonde Y, Breuer O, Lutjohann D, et al, 2014 Thyroid hormone reduces PCSK9 and stimulates bile acid synthesis in humans. J Lipid Res 55: 2408-2415. 